\section{Crossed Aldol Condensation (CAC) as a Feasible Route for Synthesis of a 1, 2-Unsaturated Carbonyl Compound-1,3 Diphenylpropenone}

\section{Abstract}

We here report an optimized crossed aldol method for synthesis of 1,2-unsaturated carbonyl compound, 1,3-diphenylpropenone (82-93\% yield) rely on alcoholic base assisted condensation of arylmethyl ketone with aromatic aldehydes. The method simply follows the conventional synthetic route however an extraordinary control over various reaction parameters enhance its synthetic practicability. Furthermore, a comparative study has been done to explore the scope for synthesis of 1,3-diphenylpropenone by crossed aldol reaction by solvent assisted method (SAM) and solvent-less method (SLM). Undeniably, solvent-less technique (90-95\% yield) hold superiority however tuning of certain reaction parameters, leveled-up solvent assisted crossed aldol reaction, but not to an analogues platform.

Keywords: Crossed aldol condensation; 1,2-unsaturated carbonyl compound; 1,3-diphenylpropenone

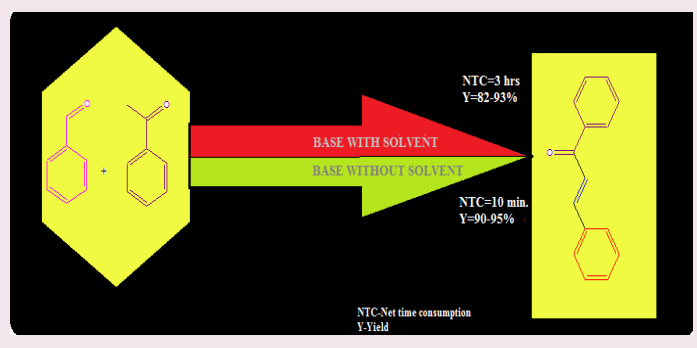

\section{Deepak Chowrasia and Nisha Sharma}

\author{
University Institute of Pharmacy, CSJM \\ University, Kanpur-208024, India
}

\section{Corresponding author:}

Deepak Chowrasia

” chowrasia.deepak@gmail.com

University Institute of Pharmacy, CSJM University, Kanpur-208024, India.

Tel: +91-9451-019049

\author{
Citation: Chowrasia D, Sharma N. Crossed \\ Aldol Condensation (CAC) as a Feasible Route \\ for Synthesis of a 1, 2-Unsaturated Carbonyl \\ Compound-1,3 Diphenylpropenone. Arch \\ Chem Res. 2016, 1:1.
}

Received: November 02, 2016; Accepted: November 11, 2016; Published: November 18, 2016

\section{Introduction}

Acrolein, acrylic acid, crotonaldehyde, crotonic acid, ethyl crotonate, isocrotonic acid, fumeric acid, maleic acid, maleic anhydride, methyl acrylate are few examples of compounds bearing 1,2-unsaturated carbonyl functionality, characterized by presence of keto-ethylenic moiety separated by a single C-C bond. This unique configuration assigns the molecule an reactive double bond amenable to get attack by nucleophile at 1 st as well 2nd position however 2 nd predominate leading to formation of 1,4 addition type product [1]. Synthetically, the 1,2-unsaturated carbonyl compounds can be synthesize by numerous synthetic pathways, aldol reaction among meritoriously distinguished. Aldol condensation represents an important class of selfcondensing reactions providing unique synthetic platform for yielding 1,2-unsaturated carbonyl compounds, further chemically modified as per applicability [2,3]. Generally, the reaction proceeds under the influence of dilute acid/base where two molecules of an aldehyde or a ketone (both containing $\alpha$-protons) condensed with each other removing water as a major side product. Crossed aldol condensation, a sister concerned aldol reaction occurs between two different carbonyl compounds (aldehyde/ketone); however, only one at this time contains $\alpha$-protons. Typically, the synthetic scope of crossed aldol reaction under general circumstances is rather more comparatively (to aldol reaction) owing to yielding multiple product at same time however efficient separation of same into desirable constituents is main commercial limitation since it potentially reduces the overall quantity of product under interest.

We here reports an optimized crossed aldol condensation and elucidate its synthetic feasibility in synthesizing 1,2-unsaturated carbonyl compound-1,3-diphenylpropenone, an important synthetic pharmacophore [4] including its practicability under 
solvent and solvent-less environment [5]. For the purpose of optimization, condensation between acetophenone and different aromatic aldehydes were done in a systematic manner with an efficient manipulation in various reaction parameters sequentially. The optimization process in addition considered factors such as prolong reaction time, excessive solvent requirement, sticky product formation, reaction failure, self condensation of enolate, side reactions, impure product, and lower yield, which were further included in the area of study. Therefore in the view of aforesaid restrictions and our own intention toward optimization of crossed aldol condensation to enhance its commercial practicability shuffled the quantity of reactants, their time frame and sequence of charging, solvent volume, amount of bases, net reaction time, overall reaction temperature and finally reframed the technique to enhance its synthetic scope.

\section{Materials and Methods}

All the commercial reagents and solvents used in this experimentation are reagent grade and were used without further treatment unless or until specified. 1H NMR spectra were recorded with a Bruker Avance II 300 NMR spectrometer. The chemical shifts were recorded in parts per million (ppm) and reported relative to the TMS. Mass spectra were recorded on Waters Q-TOF Premier-HAB213 system in ESI mode. The FT-IR spectra of synthesized compounds were recorded on PerkinElmer Spectrum version 10.03 .06. The melting point was recorded by open capillary method and is uncorrected.

\section{Experimental}

\section{Preparation of compounds by scheme 01, (1a-c)}

The 1,3-diphenylpropenone and their derivatives were synthesized in solvent (Scheme 1) and solvent-less conditions (Scheme 2) by adopting following methodology. In a clean ground neck conical flask chilled solution of sodium hydroxide (200 $\mathrm{mg}$ in $5 \mathrm{ml}$ ) was transferred followed by cold ethanol (10 $\mathrm{ml}$ ). The flask was then immersed in an ice-chest and was fitted with a sensitive thermometer, once reading of thermometer fixed at $0^{\circ} \mathrm{C}$ aromatic aldehyde $(0.01 \mathrm{M})$ was transferred at once; stirring initiated. Immediately, distilled acetophenone (0.01 M) at a rate of 5 -drops/min. was dropped in with vigorous stirring while maintaining the reaction temperature not greater than

$$
\mathrm{Ar}-\mathrm{CHO}+\mathrm{C}_{2} \mathrm{H}_{5} \mathrm{OH} \underset{\mathrm{NaOH}(10 \%)}{\stackrel{\mathrm{Ar}-\mathrm{COCH}_{3}(\text { dropwise })}{\longrightarrow}} \mathrm{Ar}-\mathrm{CH}=\mathrm{CH}-\mathrm{CO}-\mathrm{Ar}
$$

Scheme 1 For optimization and synthesis (1a-c).

$$
\mathrm{Ar}^{\prime}-\mathrm{CHO}+\mathrm{Ar}_{-}-\mathrm{COCH}_{3} \stackrel{\mathrm{NaOH} 2 \mathrm{p} \text {; Grinding }}{\mathrm{OR}} \mathrm{Ar}-\mathrm{CH}=\mathrm{CH}-\mathrm{CO}-\mathrm{Ar}
$$

$$
\mathrm{KOH}, 1 \mathrm{p} \text {, Grinding }
$$

$5^{\circ} \mathrm{C}\left(10^{\circ} \mathrm{C}\right.$ at max). Once addition was completed ice-chest was replaced with a cold water-bath $\left(15^{\circ} \mathrm{C} ; 20^{\circ} \mathrm{C}\right.$ at max) and stirring was resumed for next 3-hrs. The product so formed was filtered off, washed thoroughly initially from excess cold water followed by small quantity of chilled ethanol, and was dried at lower temperature however if required was washed separately from ethyl acetate $(10 \mathrm{ml}) .1$,3-diphenylpropenone so obtained was sufficiently pure for further usage nevertheless recrystallized from ethanol and purity regarding same was estimated from thin layer chromatography (TLC) employing ethylacetate:petroleum ether as a biphasic solvent system in a ratio of $7: 3$ (Figure 1).

\section{Preparation of compounds by scheme 02, (1a-c)}

For solvent-less technique, in a clean pestle mortar equimolar quantity $(0.01 \mathrm{M})$ of acetophenone and aromatic aldehyde were placed at room temperature. The reactant mixture was triturated in the presence of $\mathrm{NaOH}$ (2-pellet) or $\mathrm{KOH}$ (1-pellet) for at least 7-10 minutes to yield the product. The product so obtained was sufficiently pure for further usage however was recrystallized by ethanol.

1,3-diphenylpropenone (1a): Light yellowish color solid; $\mathrm{C}_{15} \mathrm{H}_{12} \mathrm{O}$ : m.p.:57-59; yield 82\%; IR ( $\mathrm{KBr}) \mathrm{cm}^{-1}$ : $3030(\mathrm{CH}-\mathrm{Ar}), 1558(\mathrm{C}=\mathrm{O})$, $1598(\mathrm{C}=\mathrm{C})$; NMR $(\delta, \mathrm{ppm}): 7.7(\mathrm{~d} 1 \mathrm{H \alpha}) 8.0(1 \mathrm{H} \beta)$ 7.4-7.9 (m, 10H-Ar-H); Mass ESI (m/e): 208.

(E)-3-(4-methoxyphenyl)-1-phenylprop-2-en-1-one (1b) Whiteyellowish color solid; $\mathrm{C}_{16} \mathrm{H}_{14} \mathrm{O}_{2}$ : m.p.:77-79; yield 89\%; IR ( $\mathrm{KBr}$ ) $\mathrm{cm}^{-1}: 2955$ (CH-Ar), 1658 (C=O), $1598(\mathrm{C}=\mathrm{C}), 1111\left(-\mathrm{OCH}_{3}\right)$; NMR

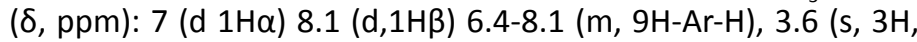
$\left.-\mathrm{OCH}_{3}\right)$; Mass ESI (m/e): 238.

(E)-3-(4-dimethylaminophenyl)-1-phenylprop-2-en-1-one (1c): Red color solid; $\mathrm{C}_{17} \mathrm{H}_{17} \mathrm{NO}$ : m.p.:110-112; yield 93\%; IR $(\mathrm{KBr}) \mathrm{cm}^{-1}$ : 2906 (CH-Ar), 1661 (C=O), 1599 (C=C), 1313 (CN); NMR (ס, ppm): 6.9 (d $1 \mathrm{H \alpha}) 8.0(\mathrm{~d}, 1 \mathrm{H} \beta)$ 7.0-7.5 (m, 9H-Ar-H), $2.6\left(\mathrm{~s}, 6 \mathrm{H},-\mathrm{N}\left(\mathrm{CH}_{3}\right)_{2}\right.$; Mass ESI (m/e): 251.

\section{Results and Discussion}

The crossed aldol condensation was successfully optimized (Table 1 and Figure 2 ) to elucidate its synthetic utility especially for 1,3-diphenylpropenone as well as its two other derivatives. The procedure we herein developed and reported provides an extraordinarily command over generation and condensation of enolate (nucleophile) with subsequent electrophile hence advantageous in terms of efficiency, cost effectiveness, devoid of reaction failure, side reactions, sticky product formation, inferior product yield, and purity problem. The change in sequence
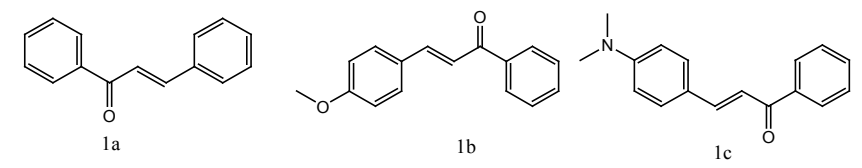

Figure 1 Structures of synthesised compounds (1,3-diphenylpropenone (1a), (E)-3-(4-methoxyphenyl)-1-phenylprop-2en-1-one(1b),(E)-3-(4-dimethylaminophenyl)-1-phenyl prop -2-en-1-one (1c). 
Table 1 Tabulation of various optimization reaction parameters.

\begin{tabular}{|c|c|c|c|c|c|c|c|}
\hline Base (NaOH) & Yield \% (1a) & Yield \% (1b) & Yield \% (1c) & Temp. $\left({ }^{\circ} \mathrm{c}\right)$ & Yield \% (1a) & Yield \% (1b) & Yield \% (1c) \\
\hline 100 & 1 & 0 & 5 & 100 & 0 & 0 & 0 \\
\hline 90 & 7 & 2 & 12 & 90 & 0 & 0 & 0 \\
\hline 80 & 19 & 17 & 27 & 80 & 0 & 2 & 0 \\
\hline 60 & 30 & 26 & 41 & 60 & 8 & 16 & 12 \\
\hline 50 & 42 & 35 & 53 & 50 & 20 & 27 & 33 \\
\hline 40 & 58 & 53 & 67 & 40 & 44 & 36 & 51 \\
\hline 30 & 66 & 70 & 79 & 30 & 64 & 68 & 71 \\
\hline 20 & 73 & 79 & 86 & 20 & 82 & 89 & 93 \\
\hline 10 & 82 & 89 & 93 & 10 & 82 & 89 & 93 \\
\hline 0 & 0 & 0 & 0 & 0 & 8 & 12 & 10 \\
\hline Solvent (ml) & Yield \% (1a) & Yield \% (1b) & Yield \% (1c) & Reaction time (min.) & Yield \% (1a) & Yield \% (1b) & Yield \% (1c) \\
\hline 100 & 32 & 30 & 32 & 20 & 0 & 0 & 0 \\
\hline 90 & 40 & 47 & 43 & 40 & 7 & 8 & 8 \\
\hline 80 & 53 & 54 & 58 & 60 & 15 & 18 & 22 \\
\hline 60 & 62 & 60 & 71 & 80 & 32 & 31 & 37 \\
\hline 50 & 71 & 69 & 73 & 100 & 45 & 50 & 47 \\
\hline 40 & 81 & 77 & 83 & 120 & 63 & 68 & 64 \\
\hline 30 & 80 & 81 & 86 & 140 & 71 & 76 & 79 \\
\hline 20 & 82 & 89 & 93 & 160 & 80 & 79 & 82 \\
\hline 10 & 82 & 89 & 93 & 180 & 82 & 89 & 93 \\
\hline 0 & 8 & 12 & 10 & 200 & 82 & 89 & 89 \\
\hline
\end{tabular}
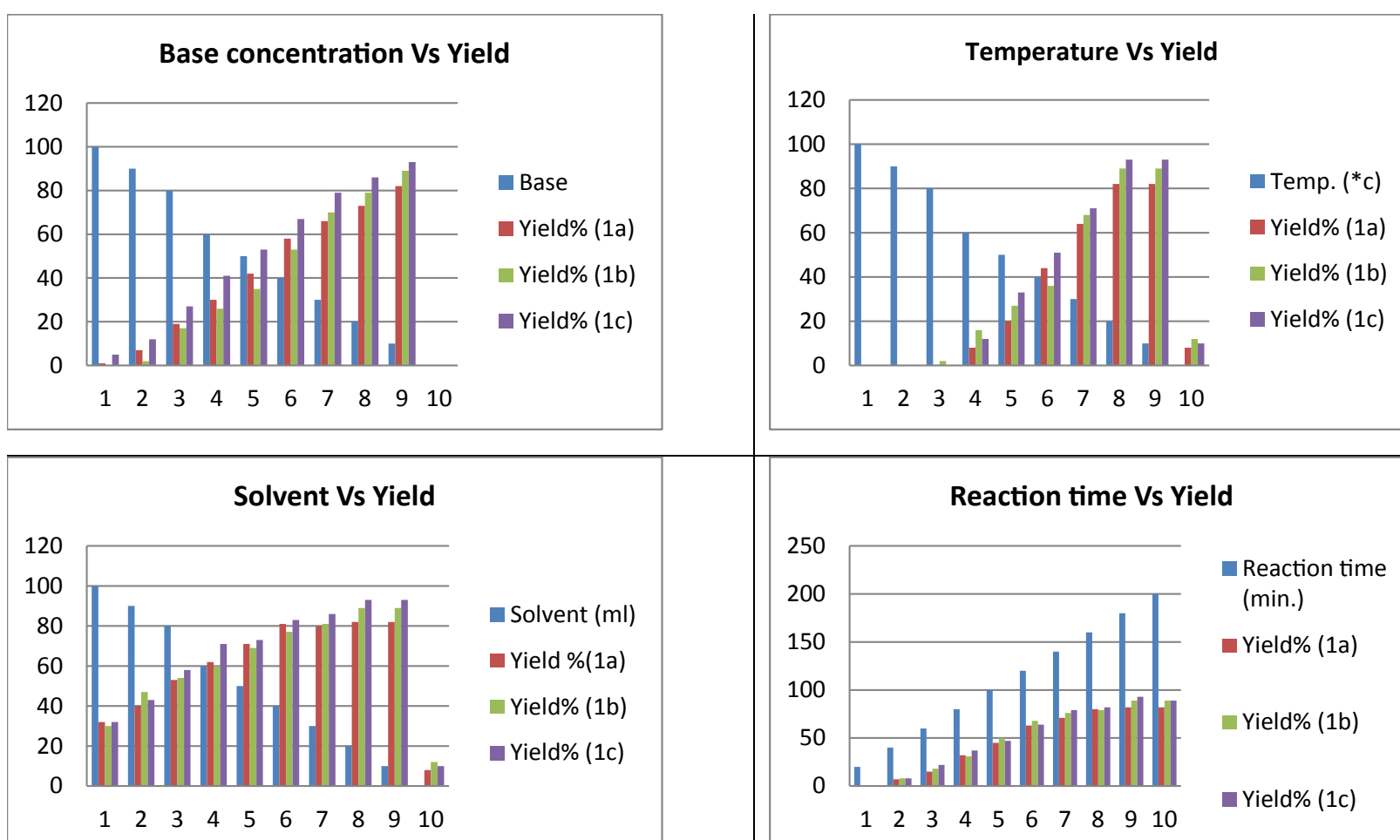

$\begin{array}{llllllllll}1 & 2 & 3 & 4 & 5 & 6 & 7 & 8 & 9 & 10\end{array}$

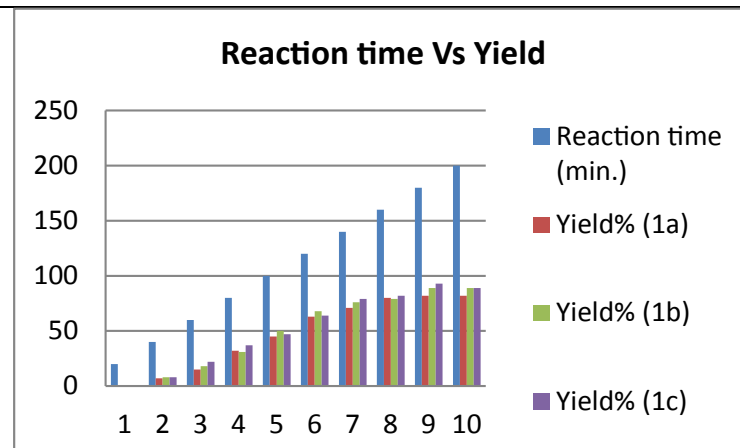

Figure 2 Statistical representation of optimized reaction parameters.

of reactant charging including their dropping rate provides an excellent control over enolate generation thus avoid selfcondensation phenomenon. We estimated that optimum drop-in rate of distilled acetophenone into the chilled reaction mixture was 5-8 drops/min whose quantity may increase up to 10-11 drops/min however any further increase in drop-in rate severely affects final product purity (sticky product), this may be assumed due to uncontrolled enolate generation and their subsequent self condensation to yield aldol type product. Feasibility of reaction at various parameters were also studied extensively, it was found that reaction proceed with its max efficiency at an initial base concentration of $10 \%$ at 10 ㅇ C which may increased up to $20 \%$ 
Table 2 Optimized reaction parameters for solvent and solventless cross-aldol condensation [5].

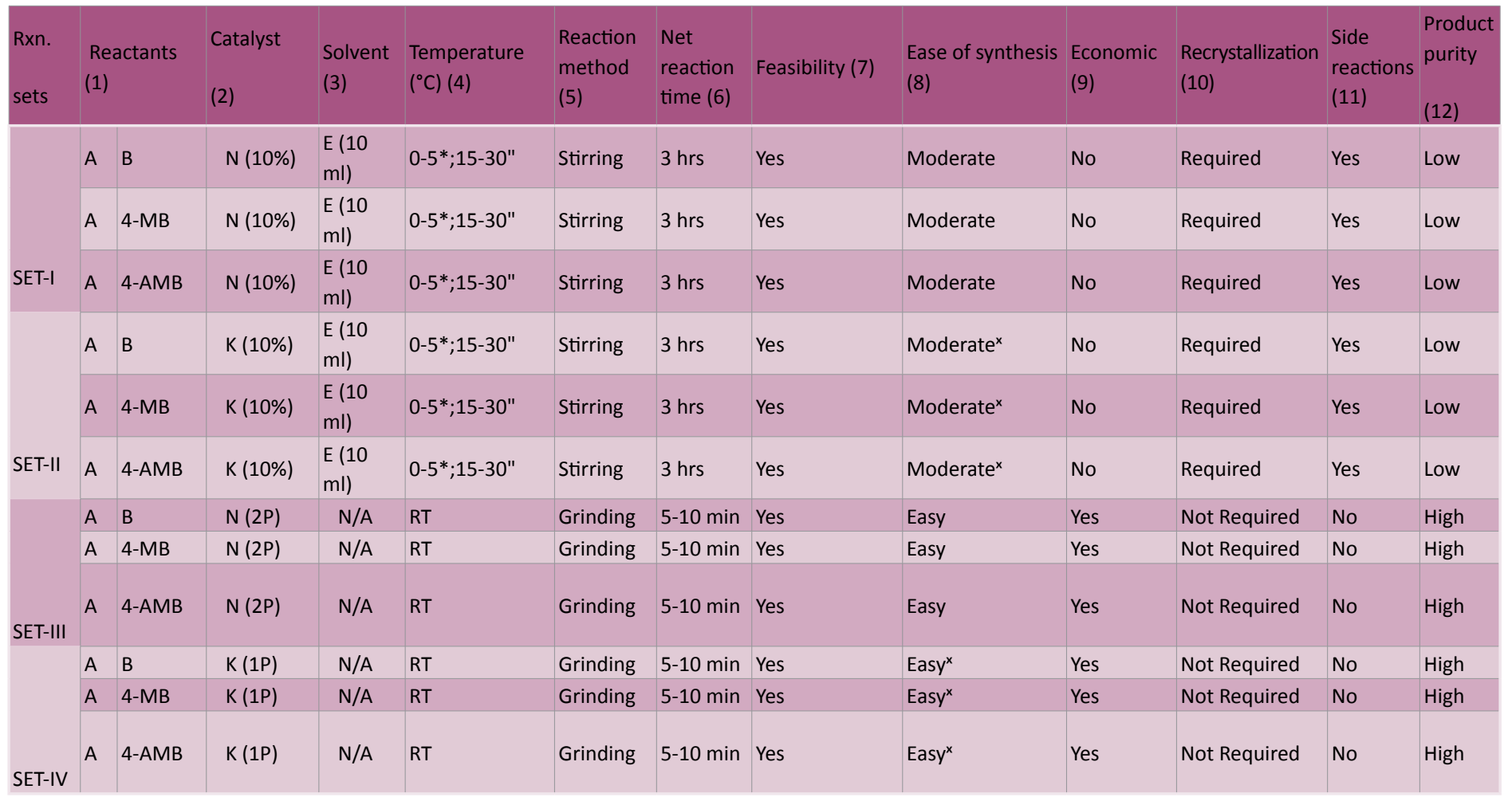

Table 3 Comparative study between SAM-solvent assisted method \& SLM-Solvent less method.

\begin{tabular}{|c|c|c|}
\hline Reaction parameters & SAM & SLM \\
\hline Reactants & $\begin{array}{l}\text { Aromatic ketone, aromatic aldehydes, and their } \\
\text { substituted derivatives }\end{array}$ & $\begin{array}{l}\text { Aromatic ketone, aromatic aldehydes, and their } \\
\text { substituted derivatives }\end{array}$ \\
\hline Reactants quantities & $0.01 \mathrm{M}$ & $0.01 \mathrm{M}$ \\
\hline Reactant charging sequence & Sequentially & Same at a time \\
\hline Reactants charging rate & $\begin{array}{c}\text { Not more than } 5 \text {-drops/min. incase aromatic } \\
\text { ketone is liquid at room temperature and a pinch } \\
\text { or slightly more if same exist as solid at room } \\
\text { temperature }\end{array}$ & Desired \\
\hline Base (Quantity and strength) & $\mathrm{NaOH}(10 \mathrm{ml} ; 10 \%)$ & $\mathrm{NaOH}$ (1-2 pallet) \\
\hline Solvent and its quantity & Ethanol; $10 \mathrm{ml}$ & Nil \\
\hline Temperature control & Strict & Room temp. \\
\hline Reaction monitoring & Required & Required \\
\hline Reaction time & $180 \mathrm{~min}$. & $10 \mathrm{~min}$. \\
\hline Side reactions & Yes & No/least \\
\hline Product Purity & Good & Excellent \\
\hline Economic & Fair & Excellent \\
\hline Eco-friendly & No & Yes \\
\hline
\end{tabular}

at $20{ }^{\circ} \mathrm{C}$ for a time limit of 180 -minutes (may up to 200 -minutes) without interfering the main product yield. At higher quantity of base $(>40 \%)$ and temperature beyond $30 \circ \mathrm{C}$ abruptly reduces net quantity of the final product under same condition, was believed due to initiation of Cannizzaro type reaction. The quantity of solvent (ethanol) used in reaction was optimized successfully, was found that $10 \mathrm{ml}$ of ethanol is sufficient to carryout condensation optimally thou may increased up to $30 \mathrm{ml}$, but quantity above this limit interferes with product precipitation. In addition synthetic feasibility of 1,3-diphenylpropenone by crossed aldol reaction under solvent assisted and solventless conditions (Table 2) were also investigated and found that later technique is superior comparatively both in terms of product purity and eco-friendly technique (Table 3). 


\section{Conclusion}

In brief, the crossed aldol condensation was successfully optimized and its various reaction parameters were revalidated to enhance synthetic scope of method for 1,2-unsaturated carbonyl compound-1,3-diphenylpropenone. In addition feasibility for the condensation of same reaction was studied in presence and absences of solvent environment whose elaborate results are given in Tables 2 and 3 respectively.

\section{Acknowledgement}

The authors gratefully acknowledge the sophisticated analytical instrumental laboratory of IIT Kanpur for IR, $1 \mathrm{H}$ NMR, and mass spectra used in this study.

\section{Competing Interest}

The authors declare no competing interests. 


\section{References}

1 Robert T (2003) Morrison, Organic Chemistry, 6 ed., Pearson/ Prentice-Hall pp: 797-809.

2 Wade LG (2006) Organic chemistry, 6 ed., Pearson/Prentice Hall p: 1262.
3 Zweifel GSN (2006) Modern Organic Synthesis: An Introduction H. Freeman \& Co Ltd p: 477.

4 Dandan X, Xie Y, Ding Y, Wu J, Hu D (2014) Molecules 19 pp: 1949119500.

5 Kakati D, Sarma JC (2011) Chemistry Central Journal 5: 8. 